\title{
Simulations of optoacoustic wave propagation in light-absorbing media using a finite-difference time-domain method
}

\author{
Deng-Huei Huang, Chao-Kang Liao, Chen-Wei Wei, and Pai-Chi Lia) \\ Department of Electrical Engineering, National Taiwan University, Taipei, Taiwan
}

(Received 21 June 2004; revised 12 December 2004; accepted 22 February 2005)

\begin{abstract}
Optoacoustic (OA) imaging is an emerging technology that combines the high optical contrast of tissues with the high spatial resolution of ultrasound. Taking full advantage of OA imaging requires a better understanding of OA wave propagation in light-absorbing media. Current simulation methods are mainly based on simplified conditions such as thermal confinement, negligible viscosity, and homogeneous acoustic properties throughout the image object. In this study a new numerical approach is proposed based on a finite-difference time-domain (FDTD) method to solve the general OA equations, comprising the continuity, Navier-Stokes, and heat-conduction equations. The FDTD code was validated using a benchmark problem that has an approximate analytical solution. OA experiments were also conducted and data were in good agreement with those predicted by the FDTD method. Characteristics of simulated OA waveforms and OA images were discussed. The simulator was also employed to study wavefront distortion in OA breast imaging. (C) 2005 Acoustical Society of America. [DOI: 10.1121/1.1893305]
\end{abstract}

PACS numbers: 43.35.Ud, 43.20.El, 43.20.Bi [AJS]

Pages: 2795-2801

\section{INTRODUCTION}

Optoacoustic (OA) effects in biological tissues have received considerable attention in recent years because of their potential applications in biomedical imaging such as depthresolved analysis of tissues, ${ }^{1}$ detection and localization of breast cancer, ${ }^{2}$ and noninvasive brain functional imaging. ${ }^{3}$ OA signals can be generated when a light-absorbing object is irradiated by modulated light. The dominant mechanism for the conversion of optical electromagnetic energy into mechanical energy in biological tissues is thermal expansion. Several algorithms have been proposed for reconstructing the internal light-absorption profile of an object, ${ }^{4-8}$ and these algorithms can generally be categorized into three modes according to the configuration of irradiation and OA signal acquisition: (1) forward mode, with the radiation source and ultrasound detection on opposite sides of the object; (2) backward mode, with the radiation source and ultrasound detection on the same side of the object; and (3) sideward mode, with ultrasound receivers perpendicular to the direction of irradiation to detect the OA signals induced inside the object. Visualization of the OA propagation in these modes, which is currently only possible via simulations, is very important to the development of effective OA imaging methods. Current simulation methods are based on simplified conditions such as thermal confinement, negligible viscosity, and homogeneous acoustic properties throughout the object to be imaged. The thermal-confinement condition is satisfied when the duration of the light pulse is much shorter than the thermal diffusion time, which is generally the case in soft tissues. The viscosity and the variations in acoustic properties, however, are not negligible for most soft tissues (e.g., blood is very viscous, and the abdomen and breast are highly inho-

\footnotetext{
a) Author to whom correspondence should be addressed. Electronic mail: paichi@cc.ee.ntu.edu.tw
}

mogeneous). In this study we start from the general OA equations that incorporate the spatial inhomogeneities of thermal and acoustic properties. A numerical approach to solving the $\mathrm{OA}$ equations is formulated using a finitedifference time-domain (FDTD) method. FDTD techniques have been used extensively to simulate the propagation and scattering of electromagnetic waves ${ }^{9}$ and acoustic waves. ${ }^{10}$ In optoacoustics, however, no similar application has yet been proposed. The approximate analytical solutions obtained by Karabutov et al. ${ }^{11}$ were then used to validate the FDTD simulator. Experimental backward OA signals from a phantom were also acquired for further validation. The characteristics of simulated OA waveforms and OA images are discussed. In addition, the simulator is employed to investigate the wavefront distortion in OA breast imaging.

In recent years considerable effort has been devoted to the study of OA effects in human breast. $^{2}$ The angiogenesis phenomenon associated with cancers makes tumors have appreciably higher optical absorption with respect to normal surrounding tissues. When such a tumor is irradiated by short laser pulses, the difference is optical absorption results in differentiable OA signals that can be used to reconstruct the optical absorption distribution. ${ }^{6,8}$ Current OA imaging algorithms, however, are based on the assumption that acoustic properties of the medium to be imaged are uniform. Unfortunately, such an assumption is rarely valid in clinical applications. Effects of acoustic heterogeneities, including acoustic impedance mismatch and sound velocity infomogeneities, on ultrasound breast imaging have long been studied by many researchers. ${ }^{12-14}$ It has been widely recognized that wavefront distortion and the resultant degradation in focusing quality is a major cause to prohibit breast ultrasound from obtaining the diffraction-limited resolution. In breast OA tomography, $\mathrm{Xu}$ and Wang ${ }^{15}$ proposed a ray approach similar to that in geometric optics to account for the OA wavefront distortion. Using acoustic ray tracing under sim- 
plified geometries, they inferred that amplitude distortion caused by refraction was not serious in breast OA tomography because OA signals are broadband, in a lower frequency range, and experience only one-way transmission distortion. However, in vitro one-way transmission experiments ${ }^{14}$ have shown that waveform distortion was generally not negligible for broadband ultrasound. In addition, full-wave approaches such as the FDTD method ${ }^{10}$ showed that waveform distortion was accumulative caused by acoustic impedance mismatch along all possible propagation paths, which cannot be fully described with simple ray tracing. An OA wavefront could potentially undergo less waveform distortion as compared to an ultrasonic wavefront because it suffers less distortion due to its lower frequency range, but it still remains unclear if the distortion is negligible in OA breast imaging because no counterparts of experiments or full-wave simulations as those in clinical breast ultrasound have yet been seen. Therefore, the simulator that we developed in this study can also be employed to study wavefront distortion in $\mathrm{OA}$ breast imaging. Numerical examples are also given in this paper to further demonstrate the usage of this FDTD simulator.

\section{MATHEMATICAL FORMULATION}

The equations of optoacoustics that relate sound excitation and the subsequent propagation in liquids to the thermal field change caused by light absorption can be formulatedwith second-order nonlinear terms and classical dissipative effects-as follows: ${ }^{16}$

$$
\begin{gathered}
\frac{\partial \rho^{\prime}}{\partial t}+\rho_{0} \nabla \cdot \vec{u}=-\rho^{\prime} \nabla \cdot \vec{u}-\vec{u} \cdot \nabla\left(\rho^{\prime}+\rho_{0}\right), \\
\rho_{0} \frac{\partial \vec{u}}{\partial t}+\nabla p=-\rho^{\prime} \frac{\partial \vec{u}}{\partial t}-\left(\rho_{0}+\rho^{\prime}\right)(\vec{u} \nabla) \bar{u} \\
+\frac{1}{3} \mu \nabla(\nabla \cdot \vec{u})+\mu \nabla^{2} \vec{u}, \\
\rho_{0} T_{0} \frac{\partial s}{\partial t}=\nabla \cdot\left(\kappa \nabla T^{\prime}\right)+W, \\
p-c_{0}^{2} \rho^{\prime}=\left(c_{0}^{2} / \rho_{0}\right)(B / 2 A) \rho^{\prime 2}+c_{0}^{2}\left(\rho \beta T / C_{\mathrm{p}}\right)_{0} s, \\
T^{\prime}-\left(T \beta / \rho C_{\mathrm{p}}\right)_{0} p=\left(T / C_{\mathrm{p}}\right)_{0} s,
\end{gathered}
$$

where $\rho^{\prime}$ is the acoustic density deviation, $\bar{u}$ is the particle velocity vector, $p$ is the acoustic pressure, $s$ is the acoustic entropy deviation, and $T^{\prime}$ is the acoustic temperature deviation. The constants $c_{0}, \rho_{0}$, and $T_{0}$ are the ambient sound speed, density, and temperature, respectively, while $\beta, \mu, \kappa$, $C_{\mathrm{p}}$, and $B / A$ are the coefficients of thermal expansion, shear viscosity, thermal conductivity, specific heat at constant pressure, and ratio of the first two terms of the Taylor series expansion of the total pressure in terms of $\rho^{\prime} . W$ is the space- and time-dependent heating function caused by light absorption. The continuity equation (1) and the NavierStokes equation (2) are derived from the laws of conservation of mass and momentum, respectively, (3) is the heatconduction equation, while (4) and (5) define the thermodynamic relations between the OA pressure and other field variables. The above equations can also be used to model acoustic propagation (without considering optoacoustic effects) in soft tissues by neglecting the heat conduction and removing the nonlinear terms and dissipative terms. ${ }^{10}$

Equations (1)-(5) can be rewritten as

$$
\begin{aligned}
& \frac{\partial}{\partial t}\left(\frac{\rho^{\prime}}{\rho_{0}}\right)+\nabla \cdot \vec{u}=N 1, \\
& \frac{\partial}{\partial t}\left(\rho_{0} \vec{u}\right)+\nabla p=N 2+D 1, \\
& \frac{\partial}{\partial t}\left(\rho_{0} T_{0} s\right)=D 2, \\
& p-c_{0}^{2} \rho^{\prime}=N 3+D 3, \\
& T^{\prime}-\left(T \beta / \rho C_{\mathrm{p}}\right)_{0} p=D 4,
\end{aligned}
$$

where

$$
\begin{aligned}
& N 1=-\left[\rho^{\prime} \nabla \cdot \vec{u}-\vec{u} \cdot \nabla\left(\rho^{\prime}+\rho_{0}\right)\right] / \rho_{0}, \\
& N 2=-\rho^{\prime} \frac{\partial \vec{u}}{\partial t}-\left(\rho_{0}+\rho^{\prime}\right)(\vec{u} \nabla) \vec{u}, \\
& N 3=\left(c_{0}^{2} / \rho_{0}\right)(B / 2 A) \rho^{\prime 2}, \\
& D 1=\frac{1}{3} \mu \nabla(\nabla \cdot \vec{u})+\mu \nabla^{2} \vec{u}, \\
& D 2=\nabla \cdot\left(\kappa \nabla T^{\prime}\right)+W, \\
& D 3=c_{0}^{2}\left(\rho \beta T / C_{\mathrm{p}}\right)_{0} s, \\
& D 4=\left(T / C_{\mathrm{p}}\right)_{0} s .
\end{aligned}
$$

In this formulation, $N 1-N 3$ denote the nonlinear terms while $D 1-D 4$ denote the dissipative terms. In general, (6)(8) [with (9) and (10) satisfied] constitute a four-dimensional (4-D; one dimension in time and three dimensions in space) problem and can be numerically solved using a FDTD method. Given the initial conditions of $\rho^{\prime}, \vec{u}$, and $s$ over the space domain, these variables (together with $p$ and $T^{\prime}$ ) can be found at all future times. However, the associated large computational and storage requirements make the 4-D scheme infeasible. One realistic scheme adopted in OA investigations is to employ an axisymmetrical cylindrical coordinate system (with the $z$-axis parallel to the direction of irradiation), which reduces the computations to threedimensional while remaining 4-D in physical nature. In subsequent sections we consider only the axisymmetrical configuration in all FDTD computations. More specifically, the two-step MacCormack scheme, ${ }^{17}$ which is fourth-order accurate in space and second-order accurate in time, was adopted to implement our finite-difference code. The first-order Mur absorbing boundary conditions ${ }^{18}$ were applied at the outer boundaries:

$$
\left[\frac{\partial f}{\partial r}-\frac{1}{c} \frac{\partial f}{\partial t}\right]_{r=r_{0}}=0,\left[\frac{\partial f}{\partial z} \mp \frac{1}{c} \frac{\partial f}{\partial t}\right]_{z= \pm z_{0} / 2}=0
$$



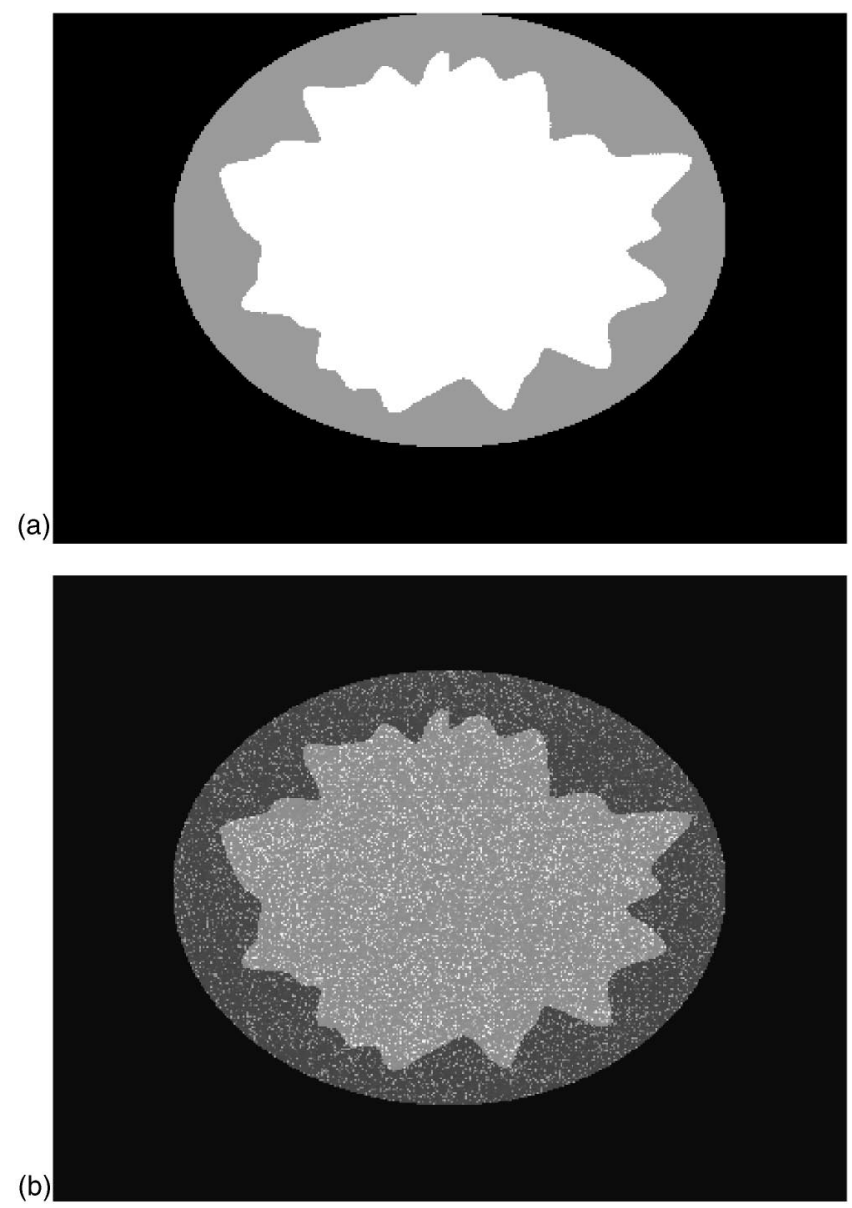

FIG. 1. Background tissue maps for the breast model. (a) Acoustic speed map. (b) Density map. Glandular parenchyma is enclosed by the irregular wall, subcutaneous fat is bounded by the irregular wall and the circular wall. Outside the circular wall is the surrounding medium used to couple the ultrasound receiver to the subcutaneous fat.

where the field parameter $f$ may be $\rho^{\prime}, \vec{u}$, or $s$, and $r_{0}$ and $z_{0}$ are the radius and length of the simulated domain, respectively. The simulation procedure is similar to that described by Sparrow and Raspet. ${ }^{19}$

The background acoustic models adopted in all breast imaging simulations were constructed according to experimental results for human breast. ${ }^{20,21}$ As shown in Figs. 1(a) and (b), the cross-sectional model consists of a zone of glandular parenchyma surrounded by subcutaneous fat. The sound speed in the parenchyma and the fat were set as 1523 and $1459 \mathrm{~m} / \mathrm{s}$, respectively. The breast model had a radius of $5 \mathrm{~cm}$. The averaged radius of the parenchyma is $4 \mathrm{~cm}$ and the thickness was varied in such a way that the arrival time fluctuations profile for an acoustic wavefront after propagating through the parenchyma wall has a root-mean-square value of $66.8 \mathrm{~ns}$ and a correlation length of $4.3 \mathrm{~mm}$, which was based on the in vitro measurements obtained by Hinkleman et al. ${ }^{21}$ Figure 1(a) illustrates one such acoustic speed model. Acoustic scattering was also incorporated in our model by adding a normally distributed, zero-mean random component with a root-mean-square value of $0.1 \mathrm{~g} / \mathrm{cm}^{3}$ to the density map in which the density of the parenchyma and the fat were set as 0.99 and $0.94 \mathrm{~g} / \mathrm{cm}^{3}$, respectively. A typical density map is shown in Fig. 1(b). The acoustic speed and average density in the surrounding medium shown in Fig. 1 were the same as those in subcutaneous fat. The configuration used in this study also had a simulated tumor inside the breast model. It had a disk with a radius of $1 \mathrm{~mm}$ placed at its center. The absorption coefficient of the disk was set to be three times that of surrounding tissues. The sound speed inside the disk was $1550 \mathrm{~m} / \mathrm{s}$ and the density was $1.12 \mathrm{~g} / \mathrm{cm}^{3}$. Simulations without heterogeneities were also performed by removing the subcutaneous fat and extending the parenchyma outside the tumors to all simulated domains.

In OA simulations, uniform irradiation was assumed with an intensity of $10 \mathrm{~mJ} / \mathrm{cm}^{2}$. The laser pulse is temporally Gaussian with a full duration at $1 / \mathrm{e}$ level of $10 \mathrm{~ns}$. Point ultrasound detectors were placed around the breast model at an interval of $0.25^{\circ}$ (i.e., totally 1440 points), while the radius of detection is $6 \mathrm{~cm}$. Received OA signals were obtained by summing the OA waveforms received at six consecutive points (i.e., totally 240 received OA signals). In pulse-echo ultrasound simulations, a linear array with a focus at the breast center was placed $1 \mathrm{~cm}$ above the breast wall to be used both as a transmitter and as a receiver. A Gaussian pulse with a central frequency of $1.5 \mathrm{MHz}$ and a 6-dB bandwidth of $1.5 \mathrm{MHz}$ was employed as the transmitted waveform. This frequency range, which was lower than usually used in breast ultrasonography but was common in breast OA imaging, was chosen to form a fair basis for the comparison between the OA wavefront and the pulse-echo wavefront. The linear array consists of 1440 points spaced at an interval of $0.043 \mathrm{~mm}$ $(\operatorname{span}=6.2 \mathrm{~cm})$. Also acoustic signals coming from six consecutive points were summed together to form 240 received acoustic waveforms.

\section{RESULTS}

The FDTD code was validated using a benchmark problem that has an analytical solution as proposed by Karabutov et al. ${ }^{11}$ The problem involves calculating the backward OA signal for a single-layer object with a uniform lightabsorption profile and acoustic properties, irradiated by a laser pulse that is Gaussian in both space and time:

$$
\begin{aligned}
p_{\text {tr }}(\vartheta= & \left.\tau / \tau_{\mathrm{L}}, z, r_{\perp}=0\right)=C \cdot\left\{A \exp \left(-A \vartheta+A^{2} / 4\right)[1\right. \\
& +\operatorname{erf}(\vartheta-A / 2)]-D \exp \left(-D \vartheta+D^{2} / 4\right)[1 \\
& +\operatorname{erf}(\vartheta-D / 2)]\},
\end{aligned}
$$

where $C$ is a scaling factor, $A=\mu_{\mathrm{a}} c_{0} \tau_{\mathrm{L}}, D=2 z c_{\mathrm{tr}} \tau_{\mathrm{L}} / a_{0}^{2}, \mu_{\mathrm{a}}$ is the light-absorption coefficient, $z$ is the normal distance between the acoustic receiver and the irradiated surface, $c_{0}$ and $c_{\text {tr }}$ are the speed of sound in the light-absorbing and transparent media, respectively, and $\tau_{\mathrm{L}}$ and $a_{0}$ are the time and radius (i.e., in the time and space domains, respectively) for the laser pulse intensity to reduce to $1 / e$ of its original level. Figure 2 shows the results for two different lightabsorption coefficients with $2 \tau_{\mathrm{L}}=9 \mathrm{~ns}$ and $D=50$. A grid spacing $(\Delta x)$ of $30 \mu \mathrm{m}$ was adopted in our FDTD code, and the grid was $1000 \times 500$. A speed of sound of $1523 \mathrm{~m} / \mathrm{s}$ was chosen for both media. The time step $\Delta t$ was set to $5 \mathrm{~ns}$ in order to meet the Courant stability condition, ${ }^{22}$ which requires that $c_{0} \Delta t / \Delta x$ should be about 0.25 to obtain the ex- 

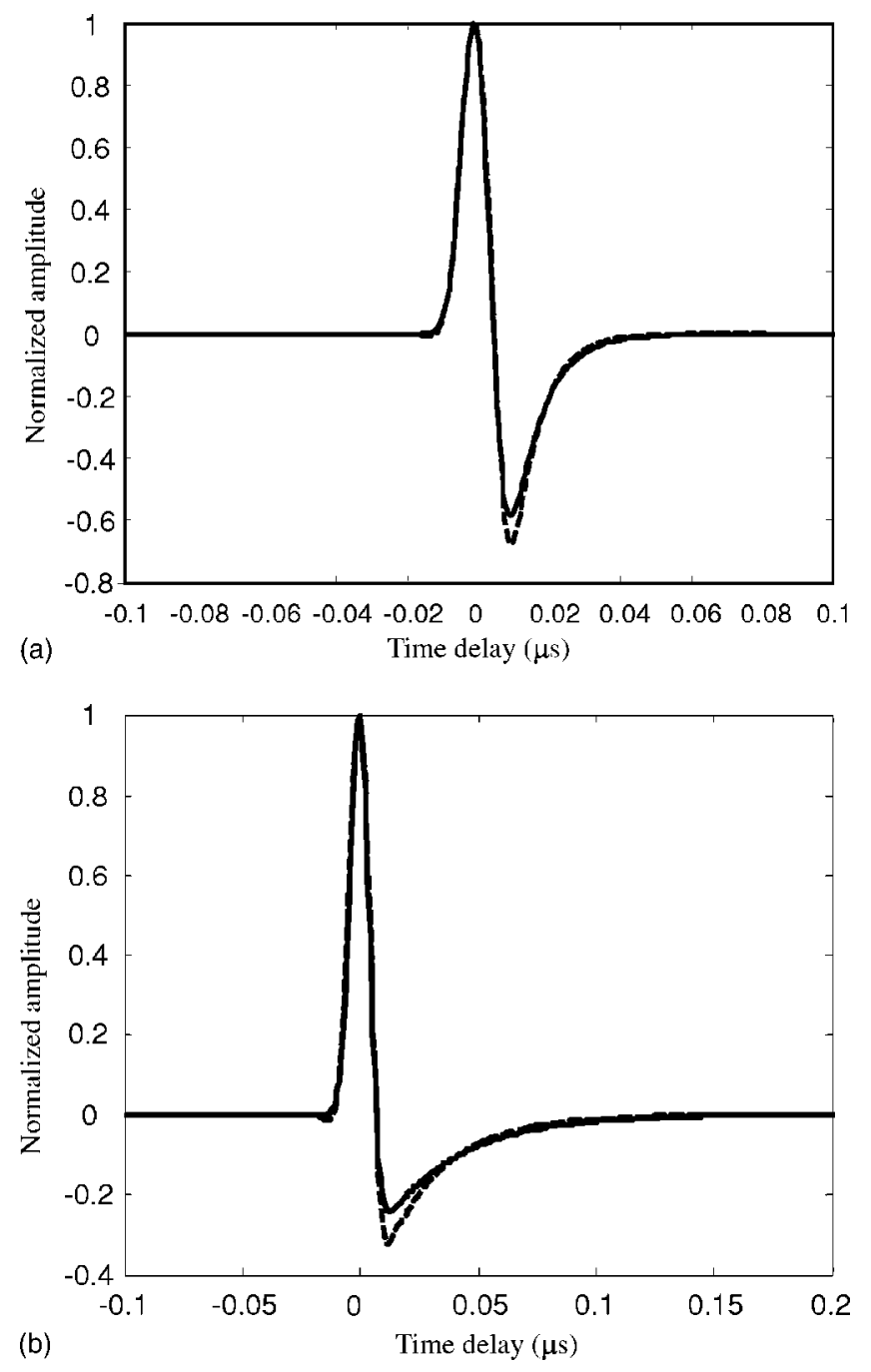

FIG. 2. Backward OA signals computed by the FDTD method (solid line) and using (19) (dashed line): (a) absorption coefficient $=160 \mathrm{~cm}^{-1}$ and (b) absorption coefficient $=600 \mathrm{~cm}^{-1}$.

pected accuracy. Figure 2(a) shows the results for $\mu_{a}$ $=160 \mathrm{~cm}^{-1}$. The solid curve refers to the OA waveform generated by the FDTD method, while the dashed curve was obtained using (19). Figure 2(b) shows the corresponding results for $\mu_{a}=600 \mathrm{~cm}^{-1}$. It can be seen that our numerical results were in excellent agreement with those from the analytical solutions; the small discrepancies were mainly attributable to the errors of the paraxial approximation used when deriving (19). ${ }^{23}$

Experimental validation was also performed using backward OA signals induced in a light-absorbing phantom comprising gelatin mixed with commercially available black pigments (OSAMA 42, SIMBALION, ROC). Pulses from a Nd:YAG laser (LS-2132U, LOTIS TII, Belarus) (10 ns at $532 \mathrm{~nm}$ ) provided irradiation with an incident energy density of $34 \mathrm{~mJ} / \mathrm{cm}^{2}$. A broadband hydrophone (GL-200, ONDA, USA) with $-3-\mathrm{dB}$ cutoff frequencies of 0.2 and $20 \mathrm{MHz}$ and high sensitivity $(28 \mathrm{nV} / \mathrm{Pa})$ was used to record the OA signals at $1 \mathrm{~cm}$ from the irradiated surface. Water was adopted as an optically transparent medium acoustically coupled to the OA phantom. The absorption coefficient was obtained by fitting (19) (i.e., the analytical solution proposed

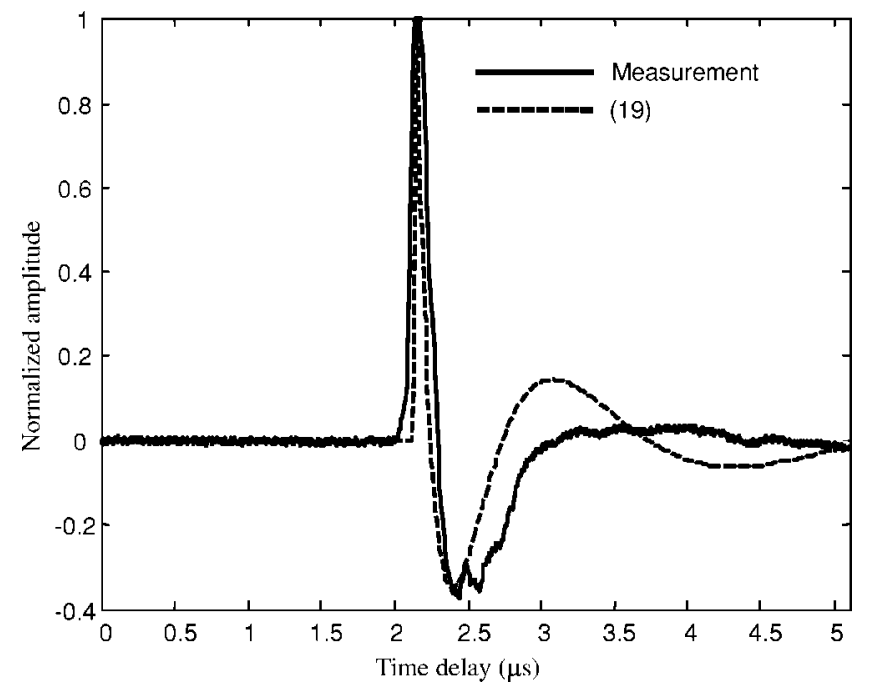

FIG. 3. Measured backward OA signal (solid line) and its curve fitting (dashed line) obtained from (19), for an absorption coefficient of $30 \mathrm{~cm}^{-1}$.

in Ref. 11) to the measured profile. The curve fitting, which was obtained by letting the measured curve and the fitted one have approximately the same amplitudes at their crests and troughs after normalization, is shown in Fig. 3 with an absorption coefficient of $30 \mathrm{~cm}^{-1}$. The solid line corresponds to the measured OA signal and the dashed line was obtained using (19) combined with a filter accounting for the impulse response of the hydrophone. The significant difference between the theoretical and experimental results is mainly attributable to (19) being less accurate at predicting extremenear-field OA signals coming from a weakly absorbing medium (i.e., $D \ll 1, A \ll 1$ ). The experimental setup, however, had $D$ at 0.011 and $A$ at 0.027 . The proposed simulator is based on the general OA equations and therefore not restricted to these limitations. It can be clearly seen in Fig. 4-in which the solid line is the measured OA signal and the dashed line was obtained using the FDTD method-that a significantly better agreement was reached. Another factor

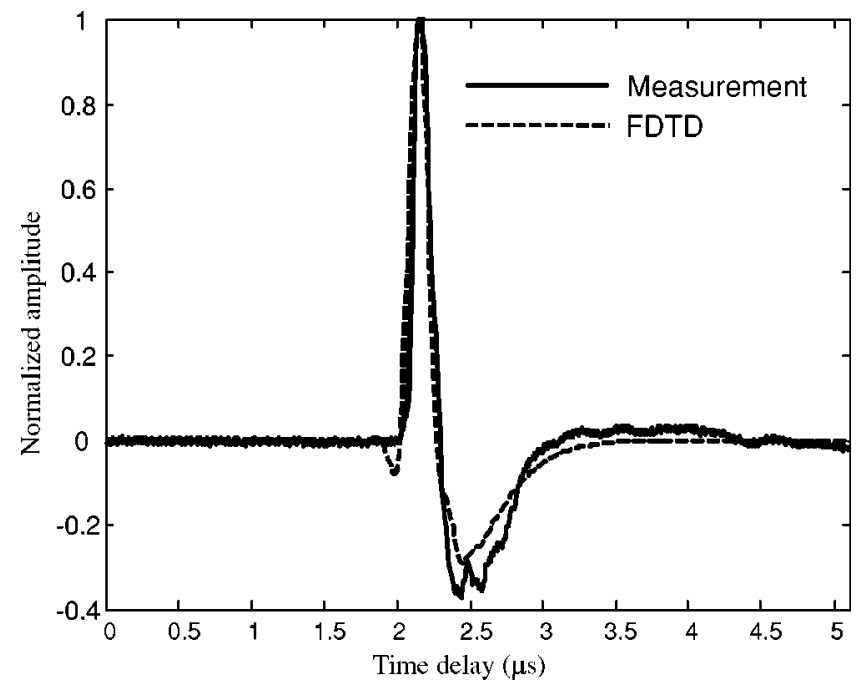

FIG. 4. Measured backward OA signal (solid line) and its curve fitting (dashed line) obtained from the FDTD method, for an absorption coefficient of $30 \mathrm{~cm}^{-1}$ and an assumed shear viscosity of $0.00086 \mathrm{~Pa}$. 


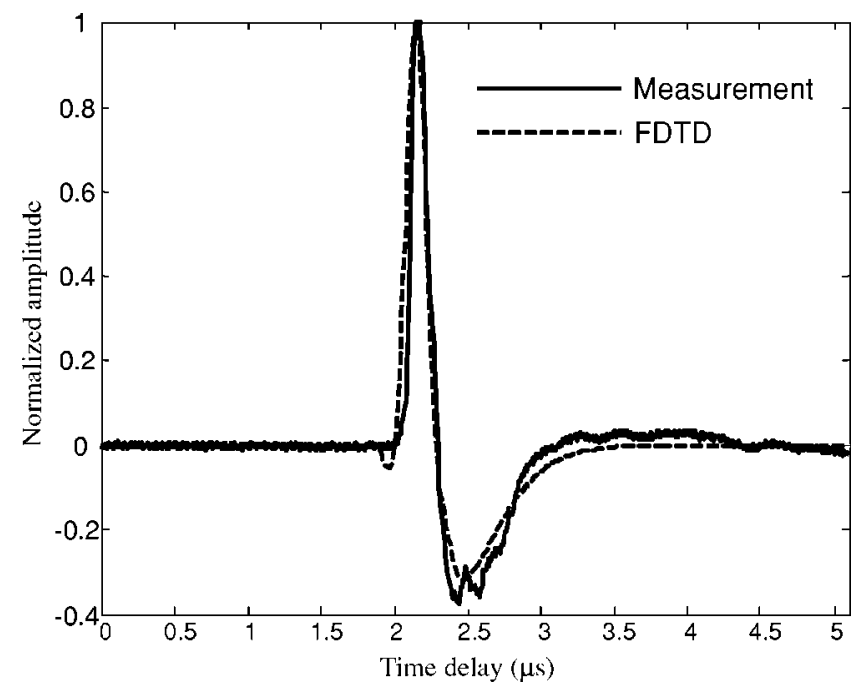

FIG. 5. Measured backward OA signal (solid line) and its curve fitting (dashed line) obtained from the FDTD method, for an absorption coefficient of $30 \mathrm{~cm}^{-1}$ and an assumed shear viscosity of $0.3 \mathrm{~Pa} \mathrm{~s}$.

not considered in (19) that would affect the OA waveform is shear viscosity. The dashed line in Fig. 5 shows the result calculated by the FDTD method with a typical shear viscosity of $0.3 \mathrm{~Pa}$ s for agar gelatin, in contrast with the result in Fig. 4 for which a low viscosity equal to that of water (0.000 $86 \mathrm{~Pa} \mathrm{~s}$ ) was used. The solid line in Fig. 5 is the same as that in Fig. 4. It can be seen that a more accurate result was obtained using high viscosity, although the improvement was minor. Moreover, our approach is more flexible and practical than the analytical approach.

A typical OA wavefield inside a homogeneous lightabsorbing object irradiated through a transparent medium using laser pulses with a Gaussian spatial intensity distribution is shown in Fig. 6. The transparent medium and the absorbing object had different acoustic properties. The distortion of the forward OA signal caused by the acoustic impedance mismatch at the interface ${ }^{23}$ and the diffraction effects on both forward and backward OA signals caused by a finite laser spot $\operatorname{size}^{23}$ are clearly visible in Fig. 6. The forward OA signal has a leading edge that suffers no shape change during propagation and can be applied directly to reconstruct the

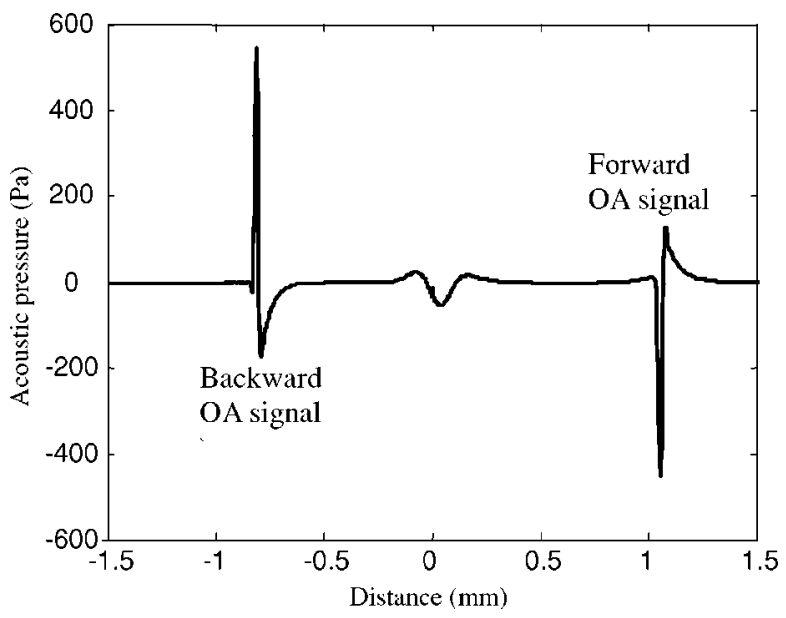

FIG. 6. OA signal profile along the axis of the laser radiation.

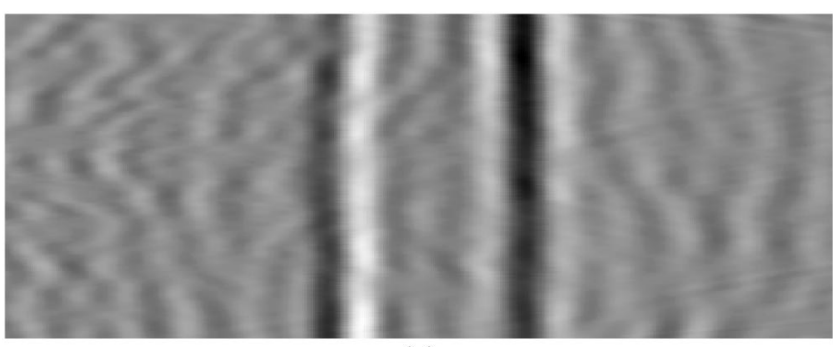

(a)

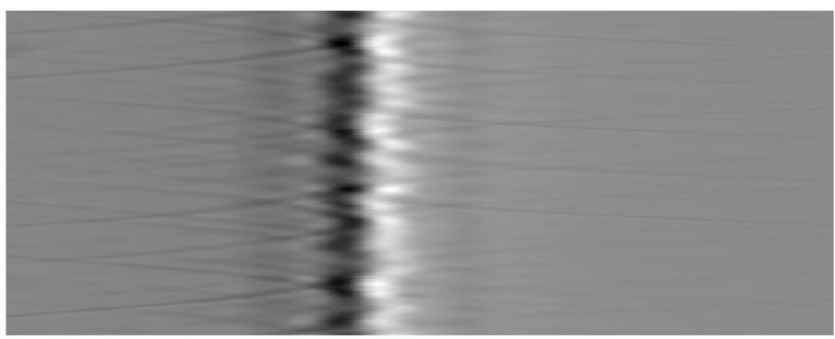

(b)

FIG. 7. (a) Pulse-echo wavefront and (b) OA wavefront for the simulated breast phantom with a tumor placed at the center. In (a), the vertical coordinate denotes the array direction and spans a distance of $6.2 \mathrm{~cm}$ in $0.26-\mathrm{mm}$ increments. In (b), the vertical coordinate denotes the receive angle extending from $0^{\circ}$ to $360^{\circ}$ with an interval of $1.5^{\circ}$. The horizontal coordinates in (a) and (b) are time with a length of $22.7 \mu$ s and an interval of $0.03 \mu \mathrm{s}$. Signal amplitude is displayed on a 128 linear gray scale, with the maximum represented by white and the minimum represented by black.

light-absorption profile. However, the forward mode requires two-sided access to the object under investigation to collect the forward OA signals, which is not practical in many clinical applications. Therefore, although backward OA signals have diffraction-dependent shapes that change with the propagation distance, they are often still used to study the absorption of optical electromagnetic energy inside a medium. ${ }^{11,24}$

Sideward OA signals have been used extensively to reconstruct the cross-sectional absorption profile ${ }^{6,8}$ inside an absorbing medium. Current reconstruction algorithms, however, are based on the assumption that the irradiated medium has uniform acoustic properties, which is rarely true in clinical situations. To elucidate the effects of acoustic heterogeneities (including the acoustic impedance mismatch and sound velocity inhomogeneities) on the reconstructed OA images, sideward OA signals were simulated. The pulse-echo acoustic wavefront and the OA wavefront formed by aligning the waveform for each scanning angle are displayed in Fig. 7. The relative-time delays between individual received waveforms in these wavefronts have been removed using the cross-correlation method. ${ }^{25}$ Obvious energy level fluctuations can be seen from both wavefronts. The pulse-echo wavefront contains two stronger subwavefronts originating from the acoustic impedance mismatch between the tumor and the parenchyma. Minor wavefronts caused by random scattering are also obvious in the pulse-echo wavefront. On the other hand, the OA wavefront consists of one main wavefront only. The corresponding OA signal and the signal suffering no acoustic heterogeneities are shown in Fig. 8 by the solid curve and dashed curve, respectively, indicating that the OA signal was appreciably distorted in pulse width and 


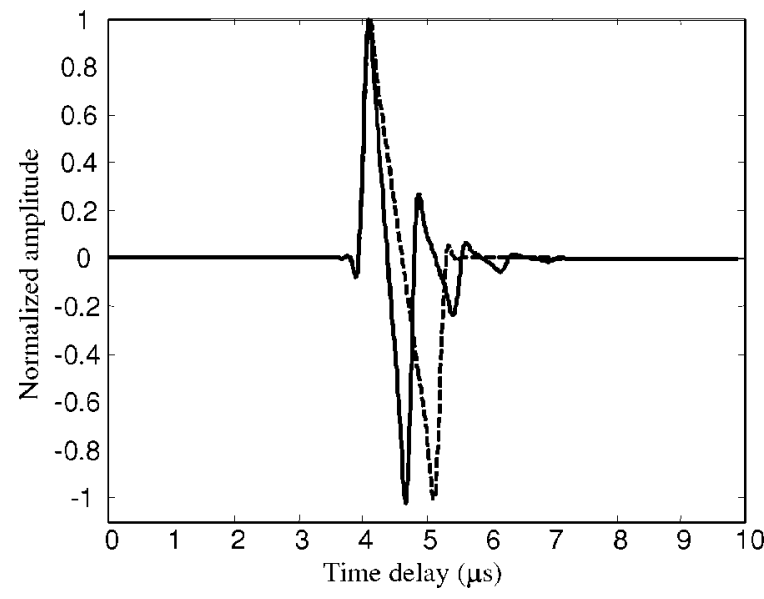

FIG. 8. Sideward OA signals for a uniformly irradiated sphere with (solid line) and without (dashed line) acoustic heterogeneities.

shape. The difference in acoustic properties between the absorbing sphere and the surrounding medium results in a series of $\mathrm{N}$-shaped waveforms in addition to the main $\mathrm{N}$-shaped one. ${ }^{26}$ Similar distortion effects, usually referred to as wavefront distortion, have been studied extensively in abdomen $^{27}$ and breast ${ }^{21}$ ultrasound images. The reconstructed absorption profile for the simulated phantom with a tumor placed at the center is shown in Fig. 9 using the timedomain algorithm proposed by Xu et al. ${ }^{8}$ Figures 9(a) and (b) show the results with and without acoustic heterogeneities, respectively. The display range is $3.3 \times 3.3 \mathrm{~cm}^{2}$. The sound speeds used in the reconstructions are $1523 \mathrm{~m} / \mathrm{s}$. It can be seen that the OA image is seriously distorted when the background tissue is acoustically inhomogeneous. This indicates that an efficient reconstruction algorithm must take acoustic heterogeneities into consideration.

\section{CONCLUSIONS}

A FDTD code has been developed to numerically solve the general $\mathrm{OA}$ equations under axisymmetrical conditions. A benchmark problem that has an approximate analytical solution was used to validate the FDTD code. Experimental validation was also performed using the OA signal from an OA phantom as measured in the backward mode. Numerical results show qualitative agreement with reported data for OA signals in the forward, backward, and sideward modes. The proposed FDTD code can be used as an aid in the design of OA imaging systems, to visual OA wave propagation in biological tissues, and to develop advanced image reconstruction algorithms.

In this study, the simulator was employed to investigate wavefront distortion in OA breast imaging. For pulse-echo ultrasound, soft tissues can be viewed as a collection of randomly distributed acoustic scatterers which result in speckle appearance in the image. On the contrary, the received wavefront in OA imaging originates from the differential thermal expansion of tissues caused by optical absorption, which is relatively a uniform property with local magnitudes that depend on the tissue types (e.g., cancerous tissue and normal tissue). Nonetheless, as demonstrated in this paper, the OA wavefront is still distorted after propagating through the

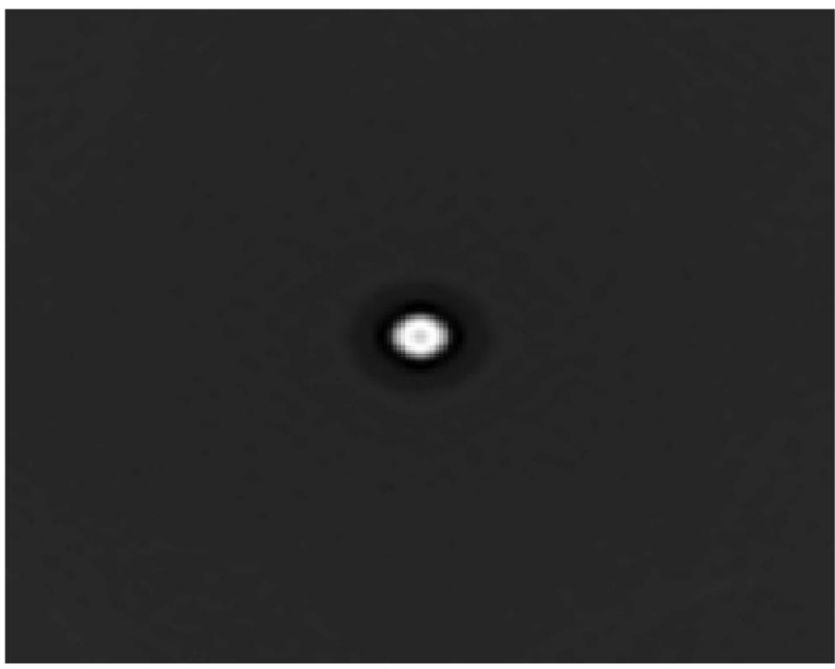

(a)

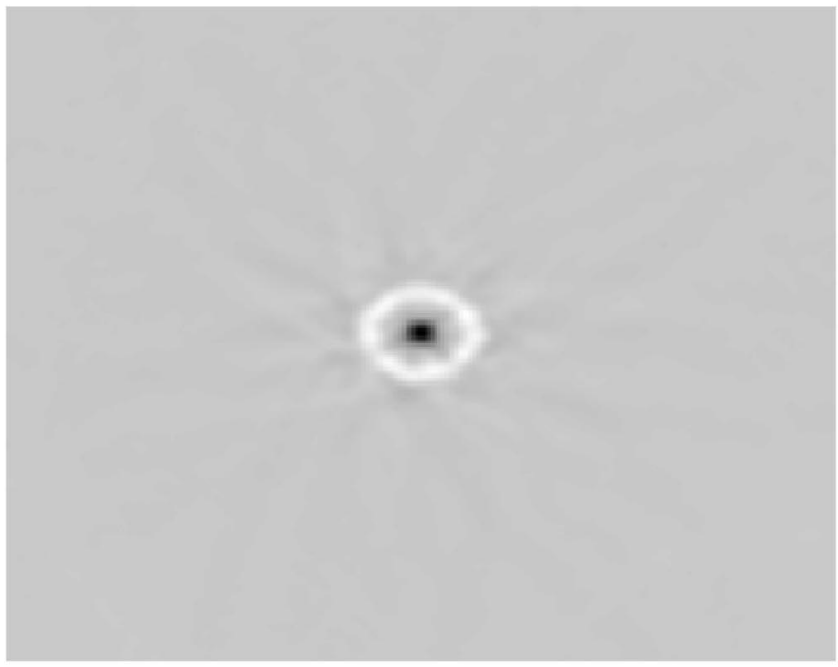

(b)

FIG. 9. Reconstructed OA images for the simulated phantom with a tumor placed at the center (a) without acoustic heterogeneities and (b) with heterogeneities. The images are displayed on a 128 linear gray scale.

breast tissue and the distortion must be corrected in order to fully realize the potential of OA imaging. In the future, the FDTD simulator described in this study will be used as an effective tool to develop and evaluate imaging algorithms that can compensate for the wavefront distortion in OA breast imaging.

${ }^{1}$ A. Beenen, G. Spanner, and R. Niessner, "Photoacoustic depth-resolved analysis of tissue models," Appl. Spectrosc. 51, 51-57 (1997).

${ }^{2}$ A. Oraevsky, A. A. Karabutov, S. V. Solomatin, E. V. Savateeva, V. G. Andreev, D. R. Fleming, Z. Gatalica, and H. Singh, "Laser optoacoustic imaging of breast cancer in vivo," Proc. Int. Soc. Opt. Eng. 4256, 6-15 (2001)

${ }^{3}$ X. Wang, Y. Pang, G. Ku, X. Xie, G. Stoica, and L. V. Wang, "Noninvasive laser-induced photoacoustic tomography for structural and functional in vivo imaging of the brain," Nat. Biotechnol. 21, 803-806 (2003).

${ }^{4}$ A. A. Oraevsky, R. O. Esenaliev, and A. A. Karabutov, "Laser optoacoustic tomography of layered tissue: Signal processing," Proc. SPIE 2979, 59-70 (1997).

${ }^{5}$ A. A. Karabutov, E. V. Savateeva, and A. A. Oraevsky, "Imaging of layered structures in biological tissues with opto-acoustic front surface transducer," Proc. SPIE 3601, 284-295 (1999).

${ }^{6}$ R. A. Kruger, P. Liu, Y. R. Fang, and C. R. Appledorn, "Photoacoustic 
ultrasound (PAUS)—Reconstruction tomography," Med. Phys. 22, 16051609 (1995).

${ }^{7}$ K. Köstli, M. Frenz, H. Bebie, and H. P. Weber, “Temporal backward projection of optoacoustic pressure transients using Fourier transform methods," Phys. Med. Biol. 46, 1863-1872 (2001).

${ }^{8} \mathrm{M}$. Xu, Y. Xu, and L. V. Wang, "Time-domain reconstruction algorithms and numerical simulations for thermoacoustic tomography in various geometries," IEEE Trans. Biomed. Eng. 50, 1086-1099 (2003).

${ }^{9}$ A. Taflove and K. R. Umashankar, "Review of FDTD numerical modeling of electromagnetic wave scattering and radar cross section," Proc. IEEE 77, 682-699 (1989).

${ }^{10}$ T. D. Mast, L. M. Hinkelman, M. J. Orr, V. W. Sparrow, and R. C. Waag, "Simulation of ultrasonic pulse propagation through the abdominal wall," J. Acoust. Soc. Am. 102, 1177-1190 (1997).

${ }^{11}$ A. A. Karabutov, E. V. Savateeva, and N. B. Podymova, "Backward mode detection of laser-induced wide-band ultrasonic transients with optoacoustic transducer," J. Appl. Phys. 87, 2003-2014 (2000).

${ }^{12}$ P. D. Freiburger, D. C. Sullivan, B. H. LeBlanc, S. W. Smith, and G. E. Trahey, "Two dimensional ultrasonic beam distortion in the breast: In vivo measurements and effects," Ultrason. Imaging 14, 398-414 (1992).

${ }^{13}$ Q. Zhu and B. D. Steinberg, "Wavefront amplitude distortion in the female breast," J. Acoust. Soc. Am. 96, 1-9 (1992).

${ }^{14}$ L. M. Hinkelman, D.-L. Liu, R. C. Waag, Q. Zhu, and B. D. Steinberg, "Measurements and correction of ultrasonic pulse distortion produced by the human breast," J. Acoust. Soc. Am. 97, 1958-1969 (1995).

${ }^{15} \mathrm{Y}$. Xu and L. V. Wang, "Effects of acoustic heterogeneity in breast thermoacoustic tomography," IEEE Trans. Ultrason. Ferroelectr. Freq. Control 50, 1134-1146 (2003).

${ }^{16}$ V. E. Gusev and A. A. Karabutov, Laser Optoacoustics (American Institute of Physics, New York, 1993), Chap. 2, pp. 45-48.

${ }^{17}$ R. W. MacCormack, Lecture Notes in Physics (Springer-Verlag, Berlin, 1971), Vol. 8, p. 151.
${ }^{18}$ G. Mur, "Absorbing boundary conditions for the finite-difference approximation of the time-domain EM field equations," IEEE Trans. Electromagn. Compat. 23, 377-382 (1981).

${ }^{19}$ V. W. Sparrow and R. Raspet, "A numerical method for general finite amplitude wave propagation and its application to spark pulses," J. Acoust. Soc. Am. 90, 2683-2691 (1991).

${ }^{20}$ G. Kossoff, E. K. Fry, and J. Jellins, "Average velocity of ultrasound in the human female breast," J. Acoust. Soc. Am. 53, 1730-1736 (1973).

${ }^{21}$ L. M. Hinkelman, D.-L. Liu, R. C. Waag, Q. Zhu, and B. D. Steinberg, "Measurements and correction of ultrasonic pulse distortion produced by the human breast," J. Acoust. Soc. Am. 97, 1958-1969 (1995).

${ }^{22} \mathrm{E}$. Turkel, "On the practical use of high-order methods for hyperbolic systems," J. Comput. Phys. 35, 319-340 (1980).

${ }^{23}$ A. A. Karabutov, N. B. Podymova, and V. S. Letokhov, "Time-resolved laser optoacoustic tomography of inhomogeneous media," J. Appl. Phys. 63, 545-563 (1996).

${ }^{24}$ K. Köstli, M. Frenz, H. Weber, G. Paltauf, and H. Kloiber, "Optoacoustic tomography: time-gated measurement of pressure distributions and image reconstruction," Appl. Opt. 40, 3800-3809 (2001).

${ }^{25}$ S. W. Flax and M. O'Donnell, "Phase aberration correction using signals from point reflectors and diffuse scatters: Basic principles," IEEE Trans. Ultrason. Ferroelectr. Freq. Control 35, 758-767 (1988).

${ }^{26}$ G. J. Diebold, M. I. Khan, and S. M. Park, "Photoacoustic signatures of particulate matter: Optical production of acoustic monopole radiation," Science 250, 101-104 (1990).

${ }^{27}$ L. M. Hinkelman, D.-L. Liu, L. A. Metlay, and R. C. Waag, "Measurements of ultrasonic pulse arrival time and energy level variations produced by propagation through abdominal wall," J. Acoust. Soc. Am. 95, 530541 (1994). 
Copyright of Journal of the Acoustical Society of America is the property of American Institute of Physics and its content may not be copied or emailed to multiple sites or posted to a listserv without the copyright holder's express written permission. However, users may print, download, or email articles for individual use. 